\title{
Ligand-Based Pharmacophore Modeling and Virtual Screening of RAD9 Inhibitors
}

\author{
Nirmal K. Prasad, ${ }^{1}$ Vishnupriya Kanakaveti, ${ }^{1}$ Siddhartha Eadlapalli, ${ }^{1}$ \\ Ramakrishna Vadde, ${ }^{1}$ Angamba Potshangbam Meetei, ${ }^{2}$ and Vaibhav Vindal ${ }^{2}$ \\ ${ }^{1}$ Department of Biotechnology \& Bioinformatics, Yogi Vemana University, Kadapa 516003, India \\ ${ }^{2}$ Department of Biotechnology, School of Life Sciences, University of Hyderabad, Hyderabad 500046, India
}

Correspondence should be addressed to Ramakrishna Vadde; vrkbtbi@yogivemanauniversity.ac.in

Received 26 May 2013; Revised 16 September 2013; Accepted 17 September 2013

Academic Editor: Marco Radi

Copyright ( 2013 Nirmal K. Prasad et al. This is an open access article distributed under the Creative Commons Attribution License, which permits unrestricted use, distribution, and reproduction in any medium, provided the original work is properly cited.

\begin{abstract}
Human RAD9 is a key cell-cycle checkpoint protein that participates in DNA repair, activation of multiple cell cycle phase checkpoints, and apoptosis. Aberrant RAD9 expression has been linked to breast, lung, thyroid, skin, and prostate tumorigenesis. Overexpression of RAD9 interacts with BCL-2 proteins and blocks the binding sites of BCL-2 family proteins to interact with chemotherapeutic drugs and leads to drug resistance. Focusing on this interaction, the present study was designed to identify the interaction sites of RAD9 to bind BCL-2 protein and also to inhibit RAD9-BCL-2 interactions by designing novel small molecule inhibitors using pharmacophore modeling and to restore BCL-2 for interacting with anticancer drugs. The bioactive molecules of natural origin act as excellent leads for new drug development. Thus, in the present study, we used the compounds of natural origin like camptothecin, ascididemin, and Dolastatin and also compared them with synthetic molecule NSC15520. The results revealed that camptothecin can act as an effective inhibitor among all the ligands taken and can be used as an RAD9 inhibitor. The amino acids ARG45 and ALA134 of RAD9 protein are interacting commonly with the drugs and BCL-2 protein.
\end{abstract}

\section{Introduction}

RAD9 is a multidomain protein, functions in multiple pathways, and is involved in the regulation of cell cycle checkpoints which contribute to genome integrity and apoptosis [1]. It promotes apoptosis by neutralizing the antiapoptotic activity of BCL-2 and BCL-xL proteins [2]. Aberrant RAD9 expression is directly linked to cancer [3]. RAD9 is evolutionarily conserved and orthologs have been isolated from a wide array of organisms, including yeast, mouse, and human [4]. RAD9 is thought to perform many of its activities as part of the RAD9-Husl-RAD1 complex [5, 6]. It was also reported that mice with a targeted homozygous deletion of RAD9 in keratinocytes are highly susceptible to the development of 7,12-dimethylbenzanthracene-induced skin tumors [7]. These studies showed that RAD9 is important for processing damaged DNA and plays a vital role in skin tumor suppressing process. Over- or underproduction of the RAD9 might have differential and more domineering effects on one subset as opposed to another of its many functions [8]. Perhaps in the context of the thyroid, prostate, breast, and lungs, the transactivity has the most profound impact on carcinogenesis and is activated when RAD9 is present at high levels $[3,9,10]$. In skin cells, underproduction of RAD9 and its impact on reducing DNA repair capacity have the greatest influence on the development of carcinogen-induced tumors [8]. Therefore, RAD9 can be viewed as a complex multifunctional protein with unique, separate domains. Because there are a large number of RAD9 interactions, it is clear that RAD9 protein is functionally complex and is an attractive target for treatment of cancer.

High expression of BCL family proteins was found in a wide variety of human cancers [11-14] and mediates the resistance of cancers to a wide spectrum of chemotherapeutic drugs and irradiation (inducing apoptosis in tumor cells) [15-17]. BCL can interfere with the therapeutic effect of many currently available anticancer drugs by blocking the cell death signals triggered by the chemotherapeutic drugs 
[18]. Therefore, the functional blockade of BCL interacting proteins like RAD9 should either restore the apoptotic process in tumor cells or sensitize these tumors for chemo- and radiotherapies. By focusing on this, the present study was designed to block the RAD9 protein interaction with BCL proteins by developing the small molecule inhibitors. By this functional blockade of RAD9, the BCL-RAD9 interactions can be prevented and make availability of BCL proteins to interact with chemotherapeutic drugs. For this small molecule, inhibitors for the active site of RAD9 (the BCL binding site) are designed by using pharmacophore modeling.

Medicinal plants derivatives are playing an important role in the treatment of cancer. Indeed, many new clinical applications of plant secondary metabolites and their derivatives are forging towards combating cancers. Many of the clinical applications have been discussed extensively by the researchers [19-22]. Pharmacophore modeling is a powerful method to rapidly identify new potential drugs. For the numerous therapeutically relevant drug targets with undetermined active site geometries, pharmacophore modeling will provides an effective mechanism for virtual screening. Various ligand-based and structure-based methods have been developed for improved pharmacophore modeling and have been successfully applied in virtual screening and lead optimization [23].

We present here the structural analysis of RAD9 and $\mathrm{BCl}-$ 2 enzymes in order to understand the protein conformations and energy minimal poses and RAD9 and BCL-2 interaction studies to understand the functional aspects followed by pharmacophore designing and ligand docking interactions to blockade the RAD9 active site.

\section{Methods}

2.1. Structural Analysis of RAD9 and BCL-2 Proteins. RAD9 structure was downloaded from Protein Data Bank (PDB ID: 3GGR, resolution $3.20 \AA$ ) [24]. The downloaded structure was energy-minimized with the SPDBV package using GROMOS 43B1 force field for further analysis. Protein sequence of human BCL-2 was retrieved from NCBI protein database (NCBI Acc. no: NP000624.2). The sequence obtained was subjected to BLASTp against PDB database to know the most appropriate structural templates. $1 \mathrm{GJH}, 1 \mathrm{O} 0 \mathrm{~L}, 3 \mathrm{OLH}$, and 2FG9 protein structures were used as templates for the construction of model. Sequence alignment of query and template was done using CLUSTALW 2.0.10 program with default parameters [25]. Modeller 9.8 was used to build the model $[26,27]$. A set of 50 models were generated, from which lower energy structure according to DOPE score was considered for further studies. Geometric inaccuracies of the structural model were calculated by subjecting the model to SAVES validating server, which validates using PROCHECK [28]. The modeled structure was further energy-minimized with the SPDBV package using GROMOS 43B1 force field. The final structure was validated again using the PROCHECK that checks for high energy regions of the modeled structure.
2.2. Docking Studies of BCL-2-RAD9. Protein-protein docking was done to study the BCL-2-RAD9 interaction by using RosettaDock server [29]. RosettaDock is a structureprediction-based program, which searches the rigid-body and side-chain conformational space of two interacting proteins to find a minimum free-energy complex structure. The docking works on multistart, multiscale Monte Carlo-based algorithm. The low-resolution phase of the search includes cycles of random rigid-body perturbations with a coursegrained representation of side chains as single pseudo atoms. The high-resolution (all atoms, including hydrogens) phase of the search includes smaller rigid-body perturbations, sidechain optimization via rotamer packing and continuous minimization, and explicit gradient-based minimization of the rigid-body displacement.

2.3. Lead Identification and Pharmacophore Generation. Ligand Scout 3.1 [30] was used to generate pharmacophore models. Training set of 20 diverse compounds was selected for lead identification to avoid any chance of correlation. The compounds were clustered according to the similarity of the pharmacophore radial distribution function (RDF). The cluster showing the highest similar compounds was selected for pharmacophore generation and changed the training set compound type to test set. The best pharmacophore model was selected on the basis of $2 \mathrm{H}$ bonds, acceptors, and optimum ADMET properties. The generated pharmacophore model was validated by checking its potential in distinguishing active from the inactive compounds in a database, the best fit values of the training set compounds, and the presence of significant chemical features required for the interaction with key catalytic residues. For the validation, an external dataset containing 5004 drug compounds was taken for RAD9 inhibition and used to validate the generated pharmacophore model.

2.4. Virtual Screening. The pharmacophore model that performed better in all the validation procedures was considered as the best pharmacophore model. It was used further as a $3 \mathrm{D}$ structural query to search chemical databases like PubChem [31], ZINC [32], and DRUGBANK [33] containing 59,652, 50,000 , and 2,38,819 compounds, respectively, in order to identify new scaffolds to be utilized in novel RAD9 inhibitor design. Searching for homologous structures against ZINC database was employed in virtual screening. Lipinski's rule of five and absorption, distribution, metabolism, elimination, and toxicity (ADMET) properties were used as primary and secondary filters to remove nondrug-like hits. In order to further refine and also to reduce the false positives of the retrieved hit compounds, molecular docking study was carried out using well-validated docking programs, namely, AutoDock [34].

2.5. Molecular Docking. The ligand and protein (RAD9) molecules were prepared in AutoDockTools. Polar hydrogens were added into the model PDB file for the preparation of protein in docking simulation. Gasteiger charge was assigned and nonpolar hydrogens were merged to the ligands. In order 
TABLE 1: Structural parameters of the model.

\begin{tabular}{lcccccccc}
\hline Protein & MHBD $(\AA)$ & MHBE & MHPh & MHPs & MCG $^{+}$ & MCG $^{-}$ & MRV $\left(\AA^{3}\right)$ & TV $\left(\AA^{3}\right)$ \\
\hline BCL-2 & 2.2 & -1.4 & -65.4 & -38.3 & -66.5 & 64.5 & 132.2 & 31592.6 \\
RAD9 & 2.2 & -1.9 & -65.1 & -36.2 & -64.9 & 67.6 & 140.7 & 37144.2 \\
\hline
\end{tabular}

MHBD: mean hydrogen bond distance; MHBE: mean hydrogen bond energy; MHPh: mean helix phi; MHPs: mean helix psi; MCG $^{+}$: mean chi gauche+; $\mathrm{MCG}^{-}$: mean chi gauche-; MRV: mean residue volume; TV: total volume (packing).

to carry out the docking simulation, AutoDock was used. It is one of the most suitable methods for performing molecular docking of macromolecules and ligands. The structures of the ligand and receptor were then saved in pqbqt format to be used for docking calculations. A three-dimensional grid box with dimensions $X=56, Y=26$, and $Z=54$ was created with grid-point spacing of $0.419 \AA$. Before running each Docking calculation, a configuration file was generated with information about grid size and coordinates and indicated the ligand and receptor files. The reports for each calculation were analyzed to obtain affinity energy $(\mathrm{Kcal} / \mathrm{mol})$ values for each ligand conformation in its respective complex. During the docking process, a maximum of 50 conformers was considered for each compound.

\section{Results and Discussions}

Investigating the relationship between RAD9 structure and function, the impact of protein-binding partners on RAD9 activity as well as how the tumor microenvironment might influence RAD9-mediated processes could be exploited to devise novel targeted therapies to treat cancer and possibly by rationally reducing or increasing RAD9 levels. This approach can be considered part of an emerging new strategy to combat cancer, where treatments are custom-designed and based on the molecular genetic profile of normal versus cancerous tissues in patients. RAD9 can function in multiple cellular processes, raising special challenges when considering the utility of ultimately targeting the protein level or activity for therapy. The RAD9 has shown to interact with BCL family proteins which are critical for resistance to chemotherapy. Thus, the RAD9 site which is prominently bound to $\mathrm{BCl}-2$ family protein is identified as a target.

The RAD9 structure retrieved from the PDB (ID: 3GGR) was subjected to energy minimization. Energy minimization was performed with Swiss-Pdb software by using GROMOS 43B1 force field. This force field allows evaluating the energy of a structure as well as repairing distorted geometries through energy minimization. The geometry of the BCL-2 model was evaluated with Ramachandran's plot calculations computed with the PROCHECK program (Figure 1). This result revealed $94.4 \%$ of the residues that were in the allowed regions and 3.6\% in generously allowed region. The modeled BCL-2 and RAD9 structures are depicted in Figure 2. Structural parameters of RAD9 and BCL-2 are shown in Table 1.

\subsection{BCL-2 Interaction with RAD9 Studies (Protein-Protein} Interaction). BCL-2 protein has shown to interact with ARG45, ARG109, SER116, ALA134, GLN210, and GLY244 (Figure 3). By comparing these interactions with those of the

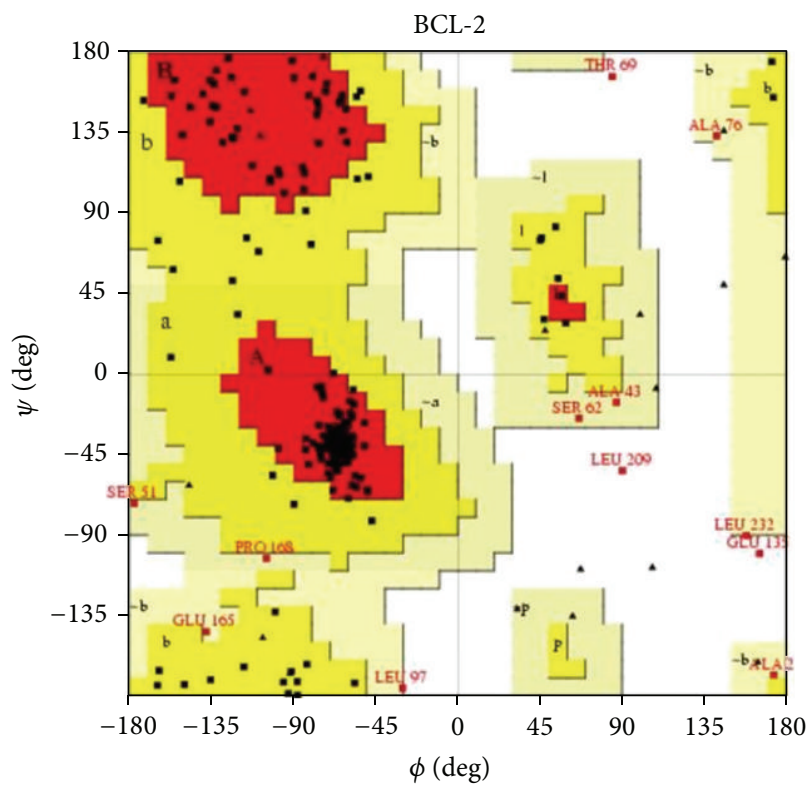

FIGURE 1: BCL-2 protein amino acids distribution in Ramachandran plot.

ligand of natural origin, we can easily consider which drug efficiently inhibits the active pocket of RAD9, preventing its interaction with BCL-2. The docking results are depicted in Table 2 .

\subsection{Lead Identification and Validation}

3.2.1. Dataset Collection. In a computerized pharmacophore generation process, the accurate choice of the training set (rough data of drugs) is a key issue. The built pharmacophore hypothesis can be as good as the input data information. The following criteria should be considered during the selection of data set in order to achieve a significant pharmacophore hypothesis. All compounds used in the training set have to bind to the same receptor in roughly the same fashion. Compounds having more binding interaction with the receptor are more active than those with fewer. The most active compounds should inevitably be included in the training set and all biologically relevant data should be obtained by homogenous procedures. Every individual feature in the resulting hypotheses will invade a certain weight that is proportional to its relative contribution to biological activity.

Based on the principle of structural diversity and wide coverage of activity range, 20 compounds were carefully selected as training set compounds and the rest were used 


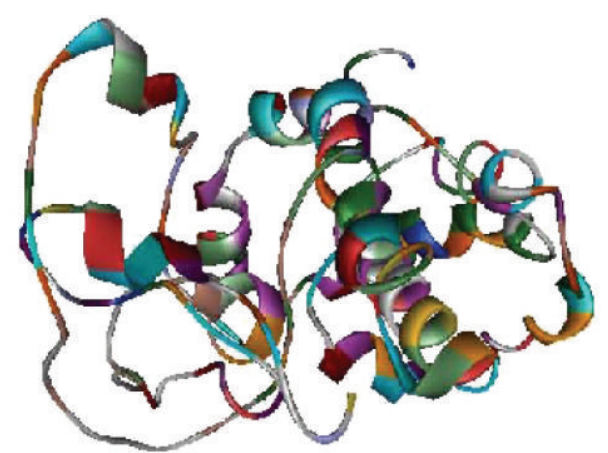

(a)

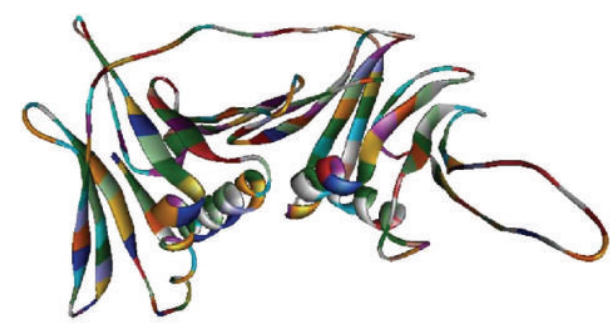

(c)

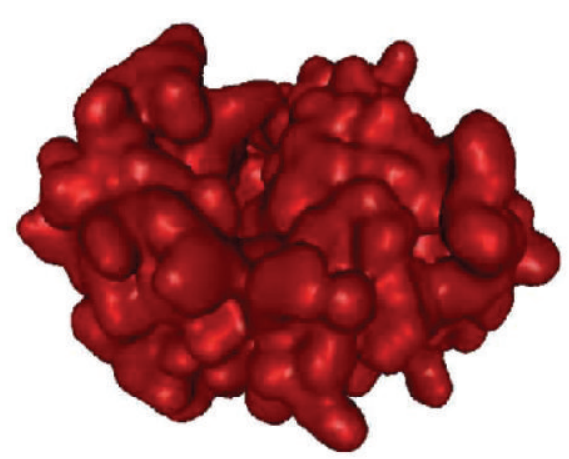

(b)

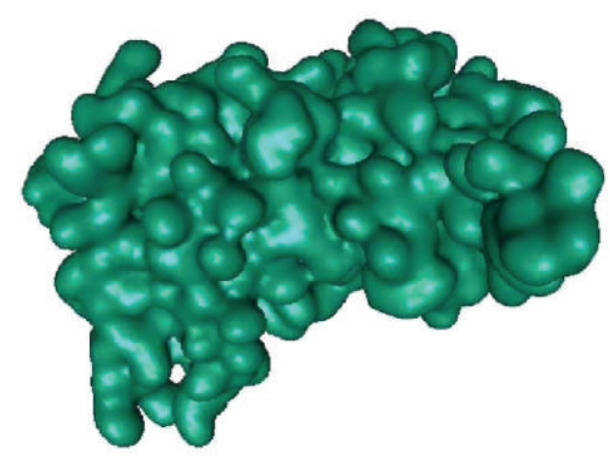

(d)

FIGURE 2: (a) Homology model of BCL-2 represented in solid ribbon pose, (b) BCL-2 model in surface representation, (c) RAD9 protein structure in solid ribbon pose, and (d) RAD9 protein structure in surface representation.

TABLE 2: Docking results of BCL-2 and RAD9 molecules.

\begin{tabular}{lccc}
\hline S.No & \multicolumn{2}{c}{ Interacting amino acids (H bond) } & Bond distance $(\AA ̊)$ \\
\hline 1 & RAD9 & BCL-2 & 2.957 \\
2 & ARG45:NH1 & GLU29:OE2 & 2.824 \\
3 & ALG45:NH2 & GLU29:OE2 & 2.833 \\
4 & GLN210:NE2 & GLY83:O & 3.132 \\
5 & GLY244:N & ASN163:OD1 & 2.945 \\
6 & ARG109:NH2 & PRO142:O & 3.109 \\
7 & SER116:OG & ASP241:OD2 & 3.048 \\
\hline
\end{tabular}

as test set in model validation. Structural and chemical features of the training set are depicted in Supplementary Table (see Supplementary Material available online at http://dx.doi.org/10.1155/2013/679459).

3.2.2. Pharmacophore Generation and Validation. Common feature pharmacophore models were built with five active training set compounds using ligand scout. The training set included homologues of camptothecin, ascididemin, dolastatin, and molecule NSC15520 chemical scaffolds. Several pharmacophore runs were carried out by changing the control parameters to develop the best model. The best pharmacophore model containing three hydrophobic and two hydrogen bond acceptor features is shown in Figure 4 along with its interfeature distance constraints. The ADMET descriptors like solubility, intestinal absorption, blood-brain barrier penetration, cytochrome P450 2D6 inhibition, and hepatotoxicity models were used. Finally, the compounds with the calculated fit value of $\geq 49$ were chosen based on the most active compound in the training set. A total of 9 pharmacophore models were generated from the best fit value showing the compound. The generated pharmacophore was subjected to screen homologous structures against ZINC database were employed. ADMET descriptor features were taken in to consideration for screening. A total of 21 compounds were obtained from virtual screening and were subjected to molecular docking study using AutoDock program to investigate the binding pose and interactions with the active site components.

3.2.3. Molecular Docking. Most active compounds in the training set and screened best hit compounds have been identified and are docked using AutoDock 4.2. Finally, four compounds, namely, camptothecin, ascididemin, dolastatin, and molecule NSC15520 were selected. In order to block the RAD9 and BCL-2 interaction, the residues on RAD9 which are interacting with the BCL-2 are taken for the docking simulations with ligands. The binding pose of all the ligand molecules at the active site of RAD9 has been analyzed. RAD9 and ligand docking statistics are depicted in Table 3. NSC15520 showed higher interacting affinity at the RAD9 active site followed by camptothecin. Binding poses 


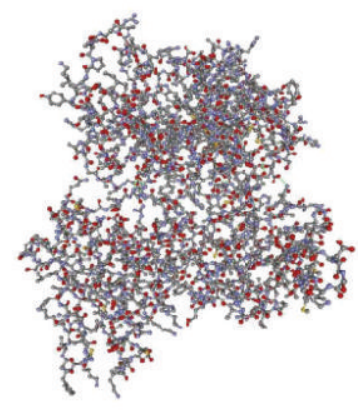

(a)

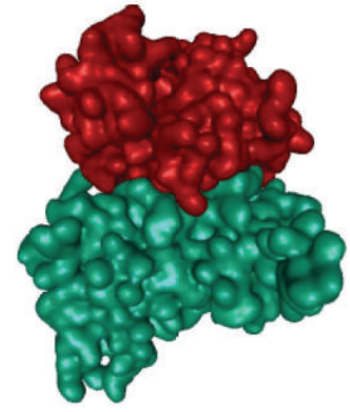

(b)

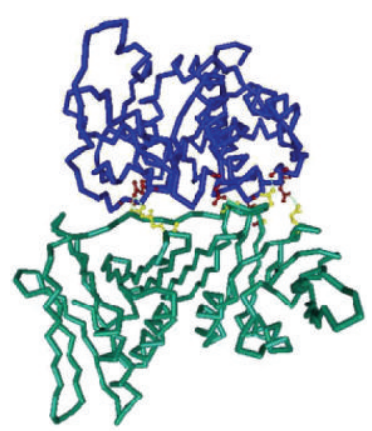

(c)

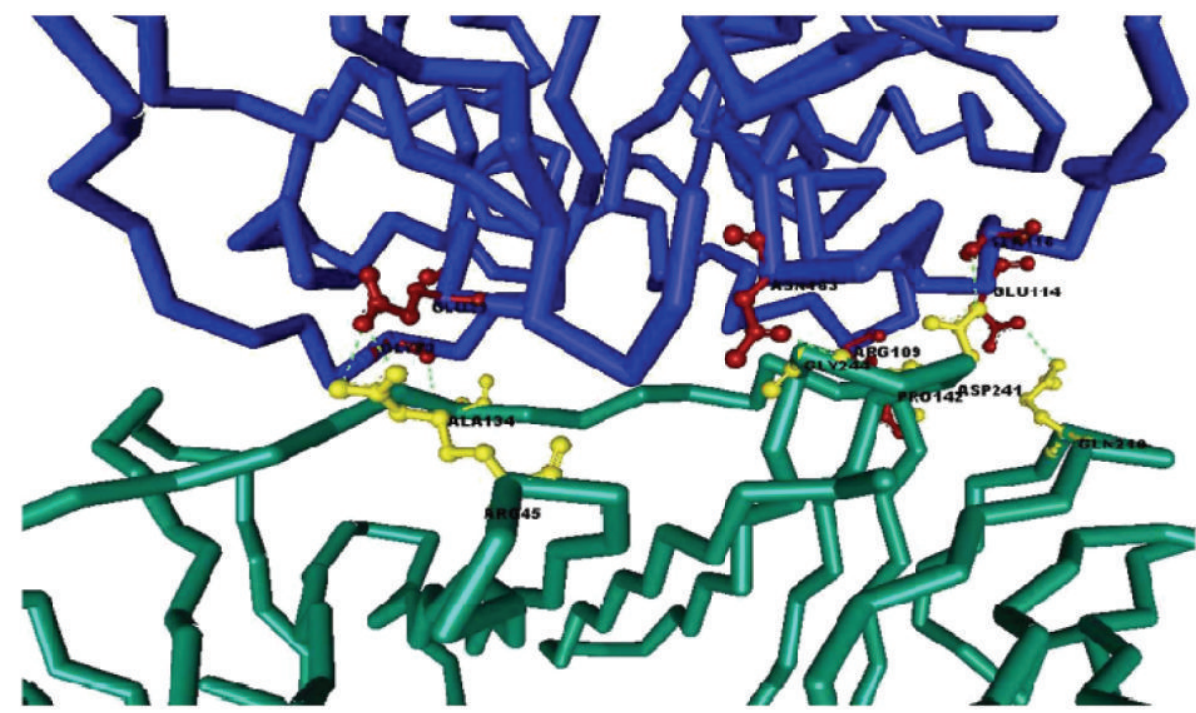

(d)

FIGURE 3: BCL-2 and RAD9 interaction represented in ball and stick (a), surface (b), sticks (c), and interacting amino acids of both proteins (d).

PHE136A

\section{ALA134A}

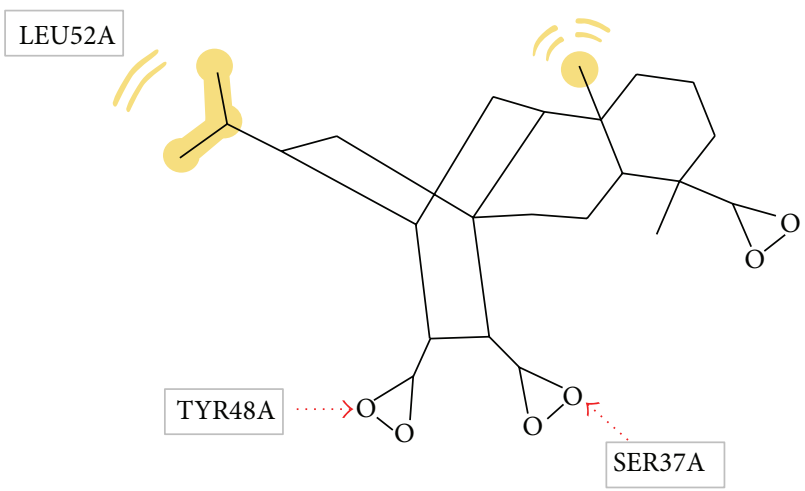

FIGURE 4: Two-dimensional spatial arrangement of the pharmacophore model showing hydrogen bonds between "SER37A and O", "TYR48A and O". Hydrogen bonds were represented by redcoloured dotted arrows and yellow colour represents hydrophobic (HY) interaction with ALA134A and PHE136A residues.
TABLE 3: RAD9 and ligands interacting statistics.

\begin{tabular}{lcc}
\hline Ligand & H-bonding residue & Bond distance $(\AA ̊)$ \\
\hline \multirow{2}{*}{ NSC15520 } & Arg39 & 2.245 \\
& Tyr48 & 1.758 \\
& Val135 & 2.018 \\
\hline \multirow{2}{*}{ Camptothecin } & Glu30 & 2.038 \\
& Val135 & 2.013 \\
\hline Dolastatin & Arg39 & 2.047 \\
\hline Ascididemin & Glu33 & 1.885 \\
\hline
\end{tabular}

of the NSC15520 and camptothecin at RAD9 active site are shown in Figure 5. The best hits of the pharmacophore are superimposed and depicted in Figure 6.

\section{Conclusion}

The data from this study demonstrate that the pharmacophore model can be used to describe the stereoselective binding of compounds at one of the sites on the RAD9 


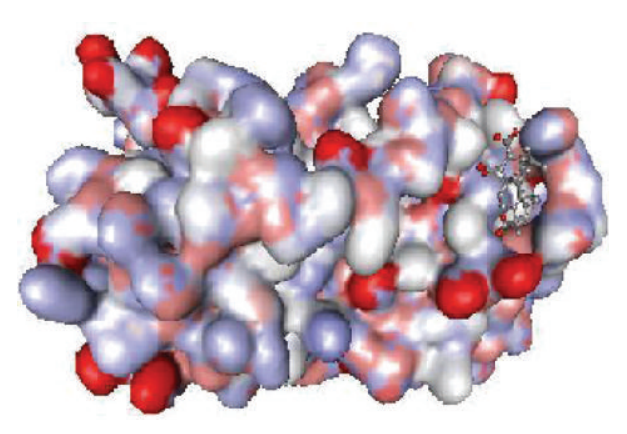

(a)

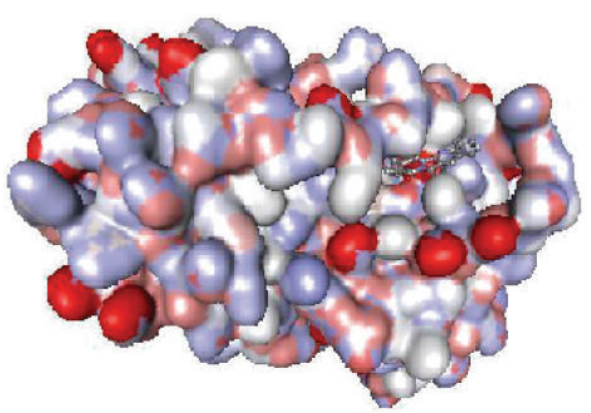

(b)

FIgURE 5: RAD9 and ligands interacting conformations (a), RAD9 and NSC15520 interacting complex, and (b) RAD9 and camptothecin interacting complex.

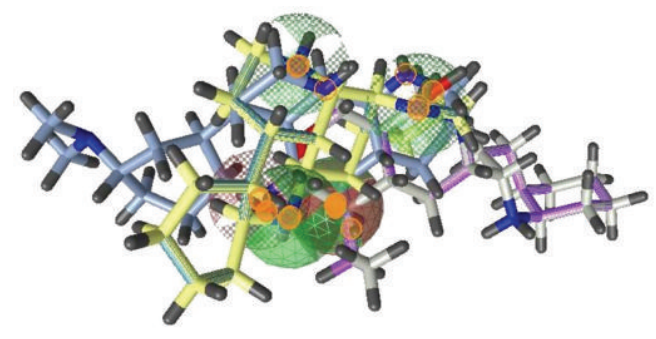

Figure 6: Superimposition of Pharmacophore and best hit compounds. Yellow coloured sticks represents shared common structural features, small Orange coloured spheres represents similar bond order and green spheres represents hydrogen bond features.

molecule. Thus, the pharmacophore model is a potential in silico screen of new drug candidates that contain chiral centres. In the present study, the compounds of natural origin curcumi, camptothecin, ascididemin, dolastatin and molecule NSC15520 were used. The results conclude that the amino acids ARG45 and ALA134 of RAD9 protein are interacting commonly with the drugs and BCL-2 protein and NSC15520 can act as an effective inhibitor among all the ligands taken.

\section{Authors' Contribution}

The authors contributed equally to the work.

\section{Acknowledgments}

The authors wish to thank Dr. Ravichand and Dr. P. V. Bramhachari for their valuable suggestions. Angamba Potshangbam Meetei is supported by the CSIR Fellowship.

\section{References}

[1] M. B. Kastan and J. Bartek, "Cell-cycle checkpoints and cancer," Nature, vol. 432, no. 7015, pp. 316-323, 2004.

[2] K. Komatsu, T. Miyashita, H. Hang et al., "Human homologue of S. pombe RAD9 interacts with BCL-2/BCL-X(L) and promotes apoptosis," Nature Cell Biology, vol. 2, no. 1, pp. 1-6, 2000.
[3] C. G. Broustas and H. B. Lieberman, "Contributions of RAD9 to tumorigenesis," Journal of Cellular Biochemistry, vol. 113, no. 3, pp. 742-751, 2012.

[4] H. B. Lieberman, "RAD9, an evolutionarily conserved gene with multiple functions for preserving genomic integrity," Journal of Cellular Biochemistry, vol. 97, no. 4, pp. 690-697, 2006.

[5] S. Y. Sohn and Y. Cho, "Crystal structure of the human RAD9Husl-RAD1 clamp," Journal of Molecular Biology, vol. 390, no. 3, pp. 490-502, 2009.

[6] M. Xu, L. Bai, Y. Gong, W. Xie, H. Hang, and T. Jiang, "Structure and functional implications of the human RAD9-Hus1-RAD1 cell cycle checkpoint complex," Journal of Biological Chemistry, vol. 284, no. 31, pp. 20457-20461, 2009.

[7] Z. Hu, Y. Liu, C. Zhang et al., "Targeted deletion of RAD9 in mouse skin keratinocytes enhances genotoxin-induced tumor development," Cancer Research, vol. 68, no. 14, pp. 5552-5561, 2008.

[8] H. B. Lieberman, J. D. Bernstock, C. G. Broustas, K. M. Hopkins, C. Leloup, and A. Zhu, "The role of RAD9 in tumorigenesis," Journal of Molecular Cell Biology, vol. 3, no. 1, pp. 39-43, 2011.

[9] Y. Maniwa, M. Yoshimura, V. P. Bermudez et al., "Accumulation of hRAD9 protein in the nuclei of nonsmall cell lung carcinoma cells," Cancer, vol. 103, no. 1, pp. 126-132, 2005.

[10] A. Zhu, C. X. Zhang, and H. B. Lieberman, "RAD9 has a functional role in human prostate carcinogenesis," Cancer Research, vol. 68, no. 5, pp. 1267-1274, 2008.

[11] S. C. Kogan, D. E. Brown, D. B. Shultz et al., "BCL-2 cooperates with promyelocytic leukemia retinoic acid receptor $\alpha$ chimeric protein (PMLRAR $\alpha$ ) to block neutrophil differentiation and initiate acute leukemia," Journal of Experimental Medicine, vol. 193, no. 4, pp. 531-543, 2001.

[12] R. Jäger, U. Herzer, J. Schenkel, and H. Weiher, “Overexpression of BCL-2 inhibits alveolar cell apoptosis during involution and accelerates c-myc-induced tumorigenesis of the mammary gland in transgenic mice," Oncogene, vol. 15 , no. 15, pp. 17871795, 1997.

[13] P. Naik, J. Karrim, and D. Hanahan, “The rise and fall of apoptosis during multistage tumorigenesis: down-modulation contributes to tumor progression from angiogenic progenitors," Genes and Development, vol. 10, no. 17, pp. 2105-2116, 1996.

[14] S. Pelengaris, M. Khan, and G. I. Evan, "Suppression of Mycinduced apoptosis in $\beta$ cells exposes multiple oncogenic properties of Myc and triggers carcinogenic progression," Cell, vol. 109, no. 3, pp. 321-334, 2002. 
[15] U. A. Sartorius and P. H. Krammer, "Upregulation of BCL-2 is involved in the mediation of chemotherapy resistance in human small cell lung cancer cell lines," International Journal of Cancer, vol. 97, no. 5, pp. 584-592, 2002.

[16] A. Ziegler, G. H. Luedke, D. Fabbro, K.-H. Altmann, R. A. Stahel, and U. Zangemeister-Wittke, "Induction of apoptosis in small-cell lung cancer cells by an antisense oligodeoxynucleotide targeting the BCL-2 coding sequence," Journal of the National Cancer Institute, vol. 89, no. 14, pp. 1027-1036, 1997.

[17] D. G. Wang, C. F. Johnston, J. M. Sloan, and K. D. Buchanan, "Expression of BCL-2 in lung neuroendocrine tumours: comparison with p53," The Journal of Pathology, vol. 184, pp. 247-251, 1998.

[18] Z. Huang, "BCL-2 family proteins as targets for anticancer drug design," Oncogene, vol. 19, no. 56, pp. 6627-6631, 2000.

[19] M. J. Balunas and A. D. Kinghorn, "Drug discovery from medicinal plants," Life Sciences, vol. 78, no. 5, pp. 431-441, 2005.

[20] D. J. Newman, G. M. Cragg, and K. M. Snader, "The influence of natural products upon drug discovery," Natural Product Reports, vol. 17, no. 3, pp. 215-234, 2000.

[21] M. S. Butler, "The role of natural product chemistry in drug discovery," Journal of Natural Products, vol. 67, no. 12, pp. 21412153, 2004.

[22] G. Samuelsson, Drugs of Natural Origin: A Textbook of Pharmacognosy, Swedish Pharmaceutical Press, Stockholm, Sweden, 5th edition, 2004.

[23] J. Fei, L. Zhou, T. Liu, and X.-Y. Tang, "Pharmacophore modeling, virtual screening, and molecular docking studies for discovery of novel Akt2 inhibitors," International Journal of Medical Sciences, vol. 10, no. 3, pp. 265-275, 2013.

[24] H. M. Berman, J. Westbrook, Z. Feng et al., "The protein data bank," Nucleic Acids Research, vol. 28, no. 1, pp. 235-242, 2000.

[25] M. A. Larkin, G. Blackshields, N. P. Brown et al., "Clustal W and Clustal X version 2.0," Bioinformatics, vol. 23, no. 21, pp. 29472948, 2007.

[26] A. Sali and T. L. Blundell, "Comparative protein modelling by satisfaction of spatial restraints," Journal of Molecular Biology, vol. 234, no. 3, pp. 779-815, 1993.

[27] N. Eswar, B. Webb, M. A. Marti-Renom et al., "Comparative protein structure modeling using MODELLER," in Current Protocols in Bioinformatics, vol. 15, pp. 5.6.1-5.6.30, Wiley, 2006.

[28] R. A. Laskowski, J. A. C. Rullmann, M. W. MacArthur, R. Kaptein, and J. M. Thornton, "AQUA and PROCHECK-NMR: programs for checking the quality of protein structures solved by NMR," Journal of Biomolecular NMR, vol. 8, no. 4, pp. 477486, 1996.

[29] S. Lyskov and J. J. Gray, “The RosettaDock server for local protein-protein docking," Nucleic Acids Research, vol. 36, pp. W233-W238, 2008.

[30] G. Wolber and T. Langer, "LigandScout: 3-D pharmacophores derived from protein-bound ligands and their use as virtual screening filters," Journal of Chemical Information and Modeling, vol. 45, no. 1, pp. 160-169, 2005.

[31] Y. Wang, J. Xiao, T. O. Suzek, J. Zhang, J. Wang, and S. H. Bryant, "PubChem: a public information system for analyzing bioactivities of small molecules," Nucleic Acids Research, vol. 37, no. 2, pp. W623-W633, 2009.

[32] J. J. Irwin and B. K. Shoichet, "ZINC-a free database of commercially available compounds for virtual screening," Journal of Chemical Information and Modeling, vol. 45, no. 1, pp. 177-182, 2005.
[33] C. Knox, V. Law, T. Jewison et al., "DrugBank 3.0: a comprehensive resource for 'Omics' research on drugs," Nucleic Acids Research, vol. 39, no. 1, pp. D1035-D1041, 2011.

[34] G. M. Morris, H. Ruth, W. Lindstrom et al., "Software news and updates AutoDock4 and AutoDockTools4: automated docking with selective receptor flexibility," Journal of Computational Chemistry, vol. 30, no. 16, pp. 2785-2791, 2009. 

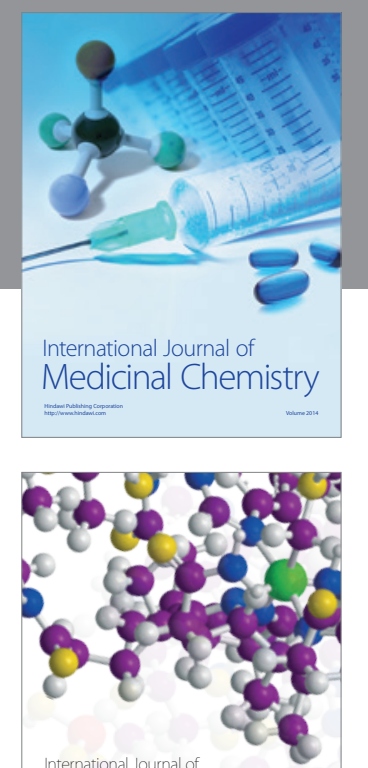

\section{Carbohydrate} Chemistry

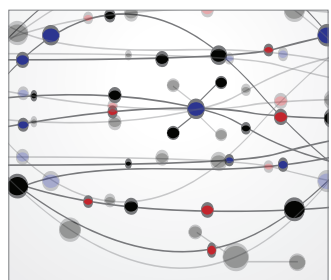

The Scientific World Journal
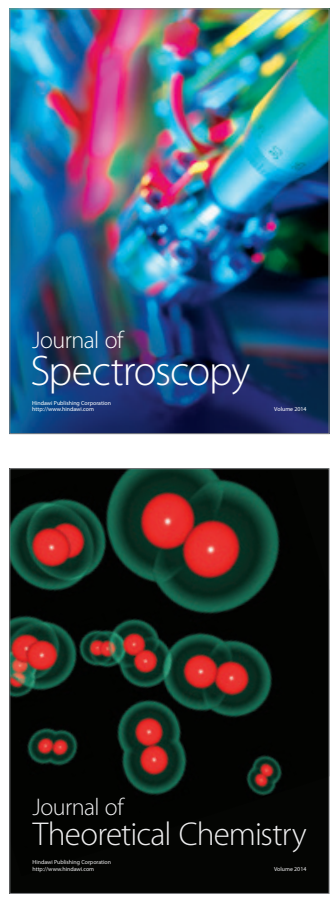
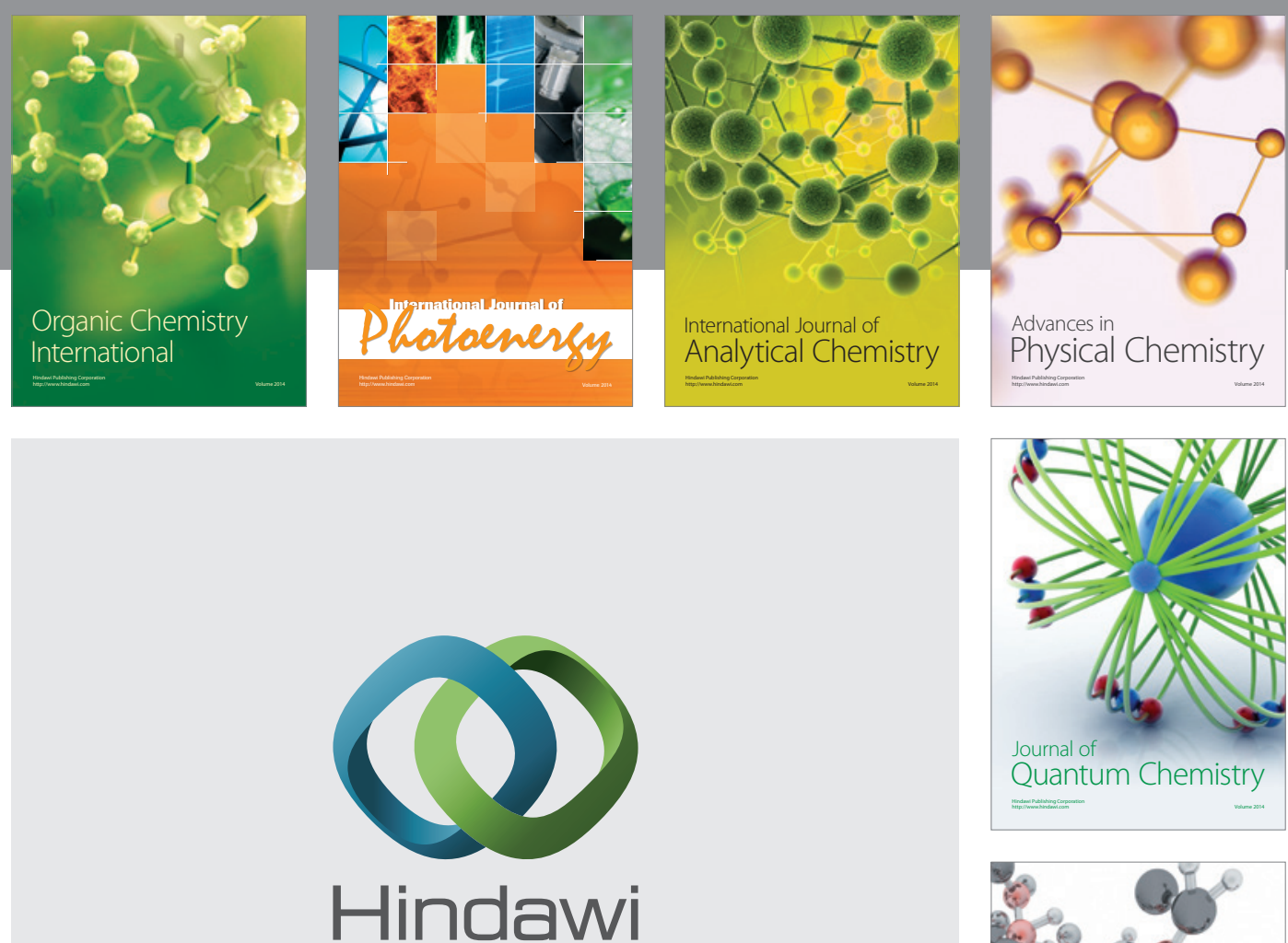

Submit your manuscripts at

http://www.hindawi.com

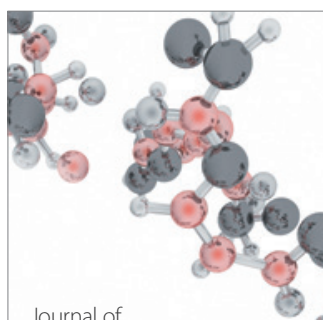

Analytical Methods

in Chemistry

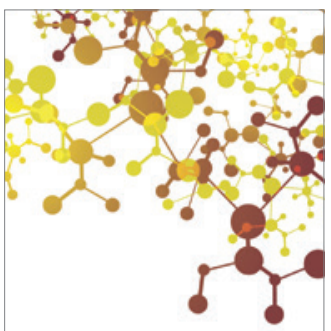

Journal of

Applied Chemistry

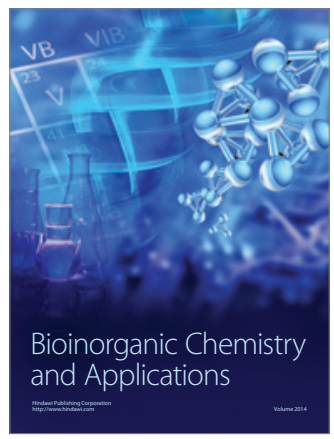

Inorganic Chemistry
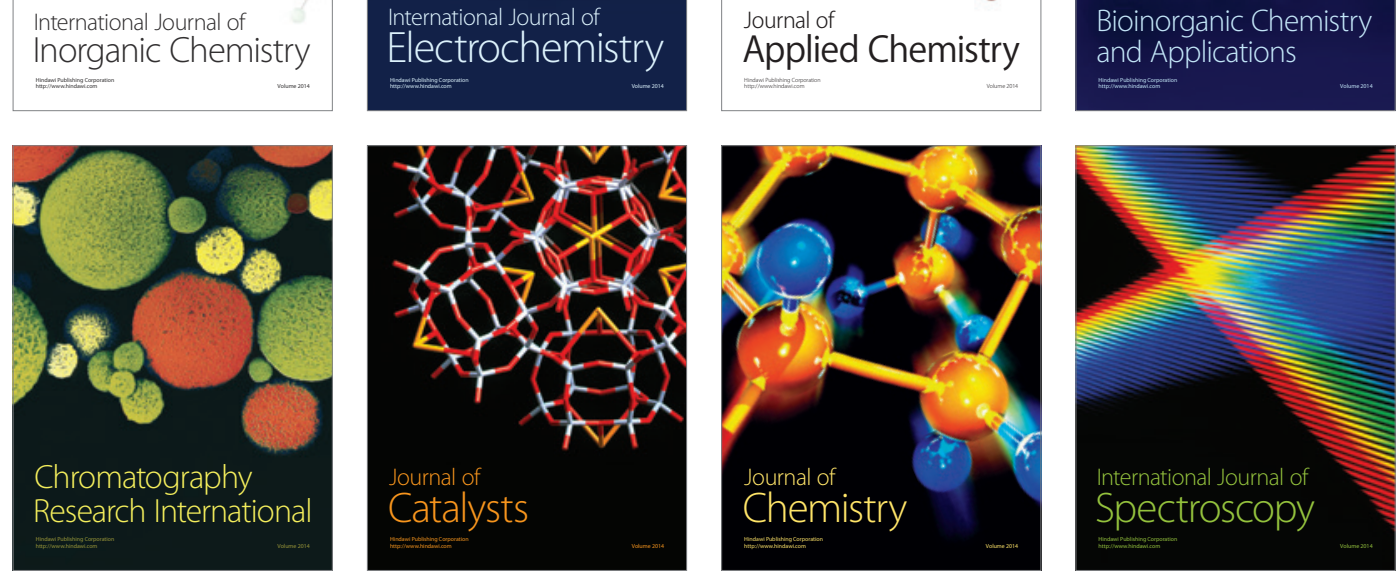